\title{
Outage Constrained Robust Beamforming Design for SWIPT-Enabled Cooperative NOMA System
}

\author{
Binbin $\mathrm{Su}^{*}$, Qiang $\mathrm{Ni}^{*}$, Wenjuan $\mathrm{Yu}^{\dagger}$, and Haris Pervaiz* \\ ${ }^{*}$ School of Computing and Communications, Lancaster University, Lancaster LA1 4WA, U.K. \\ ${ }^{\dagger}$ Institute for Communication Systems, University of Surrey, Guildford GU2 7XH, U.K.
}

\begin{abstract}
We investigate the robust beamforming design for a simultaneous wireless information and power transfer (SWIPT) enabled system, with the cooperative non-orthogonal multiple access (NOMA) protocol applied. A novel cooperative NOMA scheme is proposed, where a strong user with better channel conditions adopts power splitting (PS) scheme and acts as an energy-harvesting relay to forward the decoded signal to the weak user. The presence of channel uncertainties is considered by introducing the outage-based constraints of signal to interference plus noise ratio (SINR). Specifically, it is assumed that only imperfect channel state information (CSI) is known at the base station (BS), due to the reason that the BS is far away from both users and suffers serious feedback delay. Our aim is to maximize the strong user's data rate, by optimally designing the robust transmit beamforming and PS ratio, while guaranteeing the correct decoding of the weak user. The proposed formulation yields to a challenging nonconvex optimization problem. To solve it, we first approximate the probabilistic constraints with the Bernstein-type inequalities, which can then be globally solved by two-dimensional exhaustive search. To further reduce the complexity, an efficient lowcomplexity algorithm is proposed with the aid of successive convex approximation (SCA). Numerical results show that the proposed algorithm converges quickly, and the proposed SWIPT-enabled robust cooperative NOMA system achieves better performance than existing protocols.
\end{abstract}

Index Terms- Cooperative non-orthogonal multiple access (NOMA), simultaneous wireless information and power transfer (SWIPT), outage-based constrained optimization.

\section{INTRODUCTION}

Non-orthogonal multiple access (NOMA) has been proposed as an emerging multiple access candidate for future wireless communication systems owing to its potential to significantly improve spectral efficiency [1], [2]. Specifically, NOMA was shown to be more beneficial than conventional orthogonal multiple access (OMA) schemes in various aspects. For example, in a downlink NOMA system, the base station (BS) can send the superimposed information containing all users' messages, then the strong users obtain the prior information of the weak users, after applying successive interference cancellation (SIC) to remove the co-channel interference. The obtained prior information at the strong users can then be fully exploited with a cooperative transmission scheme, to improve the weak user's reception reliability [3].

Furthermore, since energy efficiency is another primary objective of the fifth-generation $(5 \mathrm{G})$ communications, si-

This work was supported in part by the Royal Society project IEC170324. multaneous wireless information and power transfer (SWIPT) has received considerable attention [5]. Specifically, the combination of SWIPT with NOMA has been studied by considering that NOMA users can perform energy harvesting (EH) with the received signals. For instance, a cooperative SWIPT NOMA protocol was investigated in [6], in which near NOMA users act as relays to help far NOMA users with the energy from EH instead of draining their own batteries. In [7], considering a cooperative multiple-input single-output (MISO) SWIPT NOMA system, the authors maximized the data rate of strong user ${ }^{1}$ by jointly optimizing the power splitting (PS) ratio and the beamforming precoding vectors while guaranteeing the quality of service (QoS) demand of the weak user ${ }^{2}$. However, all these studies assume that the BS has perfect knowledge of channel state information (CSI), which is quite difficult in reality due to channel estimation errors, quantization errors and feedback delay [8]. By considering a more practical scenario that the BS only knows imperfect CSI, the robust beamforming design was investigated in [9] for a MISO MC-NOMA network. The proposed design was, however, studied without involving the introduction of SWIPT, which serves as a key enabler of $5 \mathrm{G}$ and beyond.

Hence, it is natural and of great significance to investigate the transmit beamforming design in a SWIPT-enabled cooperative NOMA system with channel uncertainties considered. In existing literature, a common channel uncertainty model is the outage-based formulation which requires the outage probability of signal to interference plus noise ratio (SINR) less than a given value. In this paper, we focus on a SWIPTenabled cooperative NOMA system. Since in wireless communication systems, the downlink channel can be observed at the receiver side and fed back to the BS via an uplink control channel. Therefore, the main sources which cause imperfect CSI include channel estimation errors and/or feedback delay. Specifically, the common pilot with high transmit power is adopted in the downlink channel and shared by all receivers [10]. As a result, there is no channel estimation errors and the CSI obtained at the receiver side can be accurate. Hence we assume that all users perfectly know the CSI of downlink channels in this paper. Despite channel estimations at the user side are accurate, imperfect CSI may still result from feedback delay in the feedback link. As the users are far from the BS, the

\footnotetext{
${ }^{1}$ Here, the strong user means the user that is near the BS.

${ }^{2}$ Here, the weak user means the user that is far from the BS
} 
BS suffers serious feedback delay, which results in imperfect CSI at the BS side. On the other hand, since the two users are quite close to each other, we can assume that the feedback delay at the strong user, i.e., user 1 , is negligible. Therefore, user 1 can obtain perfect CSI of user 2, while imperfect CSI is available at the BS. We aim at designing the robust transmit beamforming to maximize the strong user's rate, based on the condition that the weak user's signal can be correctly decoded. Specifically, the contributions of this paper are summarized as follows:

- A SWIPT-enabled cooperative NOMA system is studied, with only imperfect CSI available at the BS. More specifically, the problem is formulated to optimize robust transmit beamforming design to maximize the strong user's data rate by modeling the channel uncertainties as the outage-based probability constraints. Further, in order to guarantee the correct decoding of the weak user, we require that the decoding failure probability for the weaker user's signal must fall below a given outage value.

- To solve the intractable formulation, semidefinite relaxation (SDR) technique is firstly employed to linearize the quadratic terms in the SINR expression. We then derive an efficiently computable convex approximation of the original problem with the Bernstein-type inequality method, which can be globally solved. To avoid the high complexity, the successive convex approximation (SCA)based method is proposed to iteratively solve the problem.

- Numerical results demonstrate that the proposed beamforming design is robust against channel errors compared to other schemes. Furthermore, the proposed lowcomplexity algorithm can achieve near-optimal system performance.

\section{SYSTEM MODEL}

We consider a downlink time division multiple access (TDMA) MISO transmission network, as shown in Fig. 1, wherein the $N_{t}$-antenna BS communicates with multiple users which are equipped with single antennas. There are two users in each beam and the BS performs MISO transmission with $K$ users through $M$ beams, where $K=2 M$. Since TDMA scheme is adopted for the whole system, there is no inter-cell interference between beams, we can just focus on one beam.

Let us take the first beam as an example. NOMA protocol is applied for two users. Without loss of generality, it is assumed that user 1 is a strong user and user 2 is a weak user. According to NOMA protocol, SIC is carried out at user 1, i.e., it first decodes the message of user 2 and removes user 2's message from the received signals, while user 2 treats user 1's message as noise.

Two phases are included in this SWIPT-enabled robust cooperative NOMA transmission. At the robust direct transmission phase, user 1 coordinates the process of information decoding (ID) and EH by adopting PS scheme. Specifically, as can be seen in Fig. 1, user 1 splits and directs the received signal into the information decoder and the energy harvester. As for user 2, it receives the direct

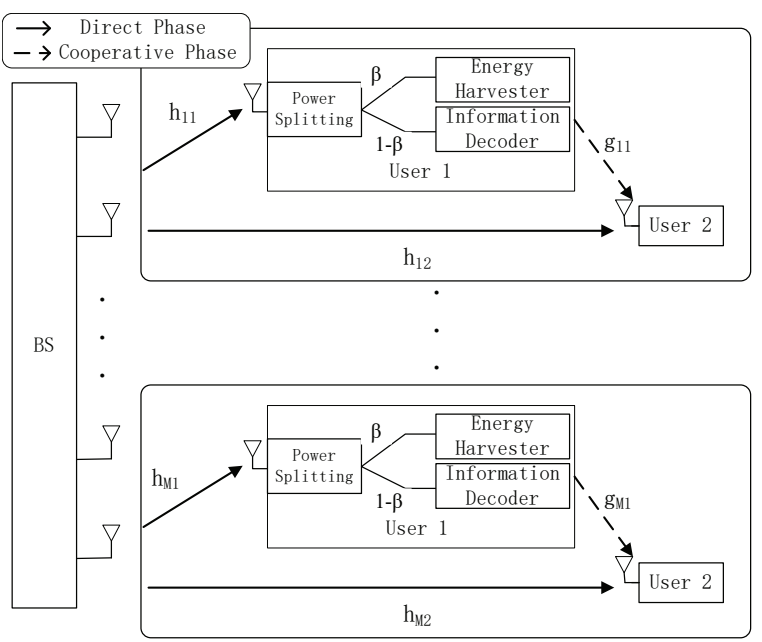

Fig. 1. System model for the SWIPT-enabled cooperative NOMA.

transmission signal from the BS at this phase. Then in the cooperative transmission phase, user 1 consumes the harvested energy to forward the decoded user 2's message to user 2 . The detailed process is summarized as follows.

\section{A. Robust Direct Transmission Phase}

During this phase, the signals for two users are superpositioned at the BS, i.e., $\boldsymbol{x}=\boldsymbol{w}_{1} x_{1}+\boldsymbol{w}_{2} x_{2}$, where $x_{1}$ and $x_{2}$ are the intended messages for the two users, and the transmit power is normalized, i.e., $\mathbb{E}\left\|x_{1}\right\|^{2}=\mathbb{E}\left\|x_{2}\right\|^{2}=1$, and $\boldsymbol{w}_{1}$ and $\boldsymbol{w}_{2}$ are the corresponding precoding vector. Then, for the weak user, i.e., user 2 , the observation is given by

$$
y_{2}^{(1)}=\boldsymbol{h}_{2}^{H}\left(\boldsymbol{w}_{1} x_{1}+\boldsymbol{w}_{2} x_{2}\right)+n_{2},
$$

where $\boldsymbol{h}_{2} \in \mathbb{C}^{N t}$ is the channel coefficient between the BS and user $2, \boldsymbol{h}_{2}^{H}$ denotes the Hermitian transpose of $\boldsymbol{h}_{2}$, and $n_{2} \sim \mathcal{C N}(0,1)$ is the additive white Gaussian noise (AWGN). In this paper, it is assumed that all channels have the same noise value as $\sigma^{2}=1$. Then the received SINR at user 2 from the direct transmission can be expressed as

$$
\operatorname{SINR}_{2}^{(1)}=\frac{\left|\boldsymbol{h}_{2}^{\mathrm{H}} \boldsymbol{w}_{2}\right|^{2}}{1+\left|\boldsymbol{h}_{2}^{\mathrm{H}} \boldsymbol{w}_{1}\right|^{2}} .
$$

Due to the assumption that there is not enough power to forward the signal of $x_{2}$ to user 2, user 1 needs to replenish the energy from the received signal based on the 'harvestthen-transmit' strategy proposed in [11]. With PS scheme employed, the received information at user 1 is given by

$$
y_{1}=\sqrt{1-\beta} \boldsymbol{h}_{1}^{H}\left(\boldsymbol{w}_{1} x_{1}+\boldsymbol{w}_{2} x_{2}\right)+n_{1},
$$

where $\beta \in[0,1]$ denotes the PS ratio, and $n_{1} \sim \mathcal{C N}(0,1)$ is the AWGN. With SIC carried out, user 1 firstly decodes the message for user 2 and then removes the information of user 2 to decode its own information. Then, the obtained SINR for 
user 1 to detect user 2's message can be expressed as

$$
\operatorname{SINR}_{1,2}=\frac{(1-\beta)\left|\boldsymbol{h}_{1}^{\mathrm{H}} \boldsymbol{w}_{2}\right|^{2}}{1+(1-\beta)\left|\boldsymbol{h}_{1}^{\mathrm{H}} \boldsymbol{w}_{1}\right|^{2}} .
$$

After removing the message of user 2 from $y_{1}$, the corresponding signal to noise ratio (SNR) of user 1 is given by

$$
\mathrm{SNR}_{1}=(1-\beta)\left|\boldsymbol{h}_{1}^{\mathrm{H}} \boldsymbol{w}_{1}\right|^{2},
$$

which will be our optimization objective in the next section.

In addition, to ensure the correct decoding ability in a given order, we have the inequality requirements [12] as below:

$$
\begin{gathered}
\left|\boldsymbol{h}_{1}^{H} \boldsymbol{w}_{2}\right|^{2} \geq\left|\boldsymbol{h}_{1}^{H} \boldsymbol{w}_{1}\right|^{2}, \\
\left|\boldsymbol{h}_{2}^{H} \boldsymbol{w}_{2}\right|^{2} \geq\left|\boldsymbol{h}_{2}^{H} \boldsymbol{w}_{1}\right|^{2} .
\end{gathered}
$$

Furthermore, with PS protocol applied at user 1 to harvest energy from the BS, the harvested energy is given as

$$
E=\zeta \beta\left(\left|\boldsymbol{h}_{1}^{H} \boldsymbol{w}_{1}\right|^{2}+\left|\boldsymbol{h}_{1}^{H} \boldsymbol{w}_{2}\right|^{2}\right) \mathrm{T},
$$

where $\zeta$ and $\mathrm{T}$ denote the EH efficiency and the transmission time fraction, respectively. Without loss of generality, we set $\zeta=1$ and $\mathrm{T}=\frac{1}{2}$ which means that equal time duration is assigned for direct and cooperative transmission stages. Hence, the available average power of user 1 can be expressed as

$$
\begin{aligned}
P_{r} & =\frac{\zeta \beta\left(\left|\boldsymbol{h}_{1}^{H} \boldsymbol{w}_{1}\right|^{2}+\left|\boldsymbol{h}_{1}^{H} \boldsymbol{w}_{2}\right|^{2}\right) T}{1-T} \\
& =\beta\left(\left|\boldsymbol{h}_{1}^{H} \boldsymbol{w}_{1}\right|^{2}+\left|\boldsymbol{h}_{1}^{H} \boldsymbol{w}_{2}\right|^{2}\right) .
\end{aligned}
$$

It is worthwhile to point out that only when user 1 can successfully detect the signals of two users received from the BS, it can then forward the signals to user 2 utilizing the harvested energy. This means that it is more important for user 1 to decode the signals than performing $\mathrm{EH}$.

\section{B. Cooperative Transmission Phase}

In the cooperative transmission phase, user 1 forwards the signal $x_{2}$ to user 2 using the harvested energy. The observation of user 2 at this phase can be characterized as

$$
y_{2}^{(2)}=\sqrt{P_{r}} g x_{2}+n_{3},
$$

where $P_{r}$ is the available power of user $1, \mathrm{~g}$ is the perfectly known channel coefficient between user 1 and user 2, and $n_{3} \sim \mathcal{C N}(0,1)$ is the normalized AWGN. The obtained SNR of user 2 at this phase is

$$
\mathrm{SNR}_{2}^{(2)}=\beta|\mathrm{g}|^{2}\left(\left|\boldsymbol{h}_{1}^{\mathrm{H}} \boldsymbol{w}_{1}\right|^{2}+\left|\boldsymbol{h}_{1}^{\mathrm{H}} \boldsymbol{w}_{2}\right|^{2}\right) .
$$

Combining the observation from both phases and using maximal ratio combination (MRC), the equivalent SINR of user 2 can be finally obtained as

$$
\begin{aligned}
\operatorname{SINR}_{2} & =\operatorname{SINR}_{2}^{(1)}+\operatorname{SNR}_{2}^{(2)} \\
& =\frac{\left|\boldsymbol{h}_{2}^{H} \boldsymbol{w}_{2}\right|^{2}}{1+\left|\boldsymbol{h}_{2}^{H} \boldsymbol{w}_{1}\right|^{2}}+\beta|g|^{2}\left(\left|\boldsymbol{h}_{1}^{H} \boldsymbol{w}_{1}\right|^{2}+\left|\boldsymbol{h}_{1}^{H} \boldsymbol{w}_{2}\right|^{2}\right) .
\end{aligned}
$$

In the next section, our aim is to maximize the achievable data rate of user 1, which is equivalent to maximize the user 1 's SNR, subject to the outage-based constraints.

\section{OUTAGE-BASED CONSTRAINED OPTIMIZATION}

In this section, the outage-based probabilistic constraints caused by imperfect CSI will be investigated, where the unsuccessful decoding of weak user falls into the scope of outage. The objective is to design robust transmit beamforming vectors $\boldsymbol{w}_{1}$ and $\boldsymbol{w}_{2}$ to maximize the achievable data rate of user 1, which is equivalent to maximize user 1's SNR while guaranteeing the outage requirements. Specifically, the outage for strong user happens when it fails to decode the weaker user's information, while for the weak user, the outage means that it can not successfully decode its own information.

The study of outage-based robust transmit optimization is a meaningful design criterion as CSI errors are prevalent in system design, and they can result in severe outage if not controlled appropriately. However, as the probability functions cannot yield straightforward closed-form expressions, how to deal with the intractable probabilistic constraints is of vital importance. To tackle the problem, we will adopt Bernstein-type inequality approach to transform the probability constraints. It has been proved in [13] that the Bernstein-type inequality can get a rank-one solution which means we can drop the rankone constraint with the application of SDR. We formulate the robust transmit beamforming design problem as follows:

$$
\begin{aligned}
& \text { P1 : } \max _{\beta, \boldsymbol{w}_{1}, \boldsymbol{w}_{2}} \quad(1-\beta)\left|\boldsymbol{h}_{1}^{H} \boldsymbol{w}_{1}\right|^{2} \\
& \text { s.t. } \quad \operatorname{Pr}\left(\mathrm{SINR}_{1,2} \geq \gamma\right) \geq 1-\rho_{1}, \\
& \operatorname{Pr}\left(\mathrm{SINR}_{2} \geq \gamma\right) \geq 1-\rho_{2}, \\
&|| \boldsymbol{w}_{\mathbf{1}}\left\|_{2}^{2}+\right\| \boldsymbol{w}_{\mathbf{2}} \|_{2}^{2} \leq P_{\max }, \\
&\left|\boldsymbol{h}_{1}^{H} \boldsymbol{w}_{2}\right|^{2} \geq\left|\boldsymbol{h}_{1}^{H} \boldsymbol{w}_{1}\right|^{2}, \\
&\left|\boldsymbol{h}_{2}^{H} \boldsymbol{w}_{2}\right|^{2} \geq\left|\boldsymbol{h}_{2}^{H} \boldsymbol{w}_{1}\right|^{2}, \\
& 0 \leq \beta \leq 1,
\end{aligned}
$$

where $\gamma$ is the target SINR of user $2, \rho_{i} \in[0,1), i=1,2$, is the maximum tolerable outage probability for two users, and $P_{\max }$ is the maximum available transmit power at the BS. Constraints (12e) and (12f) represent the decoding capability requirements [12].

To solve the problem P1, we first relax it by applying SDR approach. Specifically, we replace the beamforming vector $\boldsymbol{w}_{i}$ by semidefinite positive matrices $\boldsymbol{W}_{i}$, i.e,

$$
\boldsymbol{W}_{i}=\boldsymbol{w}_{i} \boldsymbol{w}_{i}^{H}, i=1,2 .
$$

Then, with imperfect CSI at the BS, the channel error models are assumed to be $\tilde{\boldsymbol{e}}_{h_{i}} \sim \mathcal{C N}\left(\mathbf{0}, \boldsymbol{C}_{i}\right), i=1,2$, where the given error covariance $\boldsymbol{C}_{i} \succeq \mathbf{0}$. Hence, the channels can be further denoted as:

$$
\boldsymbol{h}_{i}=\tilde{\boldsymbol{h}}_{i}+\boldsymbol{C}_{i}^{\frac{1}{2}} \boldsymbol{e}, \quad i=1,2 .
$$

where $\boldsymbol{h}_{i}$ is the practical channel gain, $\tilde{\boldsymbol{h}}_{i}$ is channel estimation at the BS and $e \sim \mathcal{C N}\left(\mathbf{0}, \boldsymbol{I}_{N_{t}}\right)$. 
By replacing $\boldsymbol{h}_{i}$ with $\tilde{\boldsymbol{h}}_{i}+\boldsymbol{C}_{i}^{\frac{1}{2}} \boldsymbol{e}$ and denoting that $\Gamma=$ $\left(\tilde{\boldsymbol{h}}_{1}+\boldsymbol{C}_{1}^{\frac{1}{2}} \boldsymbol{e}\right)^{H}\left(\boldsymbol{W}_{2}-\gamma \boldsymbol{W}_{1}\right)\left(\tilde{\boldsymbol{h}}_{1}+\boldsymbol{C}_{1}^{\frac{1}{2}} \boldsymbol{e}\right)$, the probabilistic SINR constraint (12b) can be recast as

$$
\operatorname{Pr}\left(\Gamma \geq \frac{\gamma}{1-\beta}\right) \geq 1-\rho_{1} .
$$

As the other outage-based SINR constraint (12c) has a more complicated form, we transform it by introducing an auxiliary variable $\theta$. Firstly, (12c) is decomposed into the following two sub-problems:

$$
\begin{gathered}
\operatorname{Pr}\left\{\beta|g|^{2}\left(\left|\boldsymbol{h}_{1}^{H} \boldsymbol{w}_{1}\right|^{2}+\left|\boldsymbol{h}_{1}^{H} \boldsymbol{w}_{2}\right|^{2}\right) \geq \gamma-\theta\right\} \geq 1-\rho_{2}, \\
\frac{\left|\boldsymbol{h}_{2}^{H} \boldsymbol{w}_{2}\right|^{2}}{\left|\boldsymbol{h}_{2}^{H} \boldsymbol{w}_{1}\right|^{2}+1} \geq \theta,
\end{gathered}
$$

where the optimality of the decomposition can be assured when (16b) holds with equality. It is easy to notice that (16a) has the same form as (12b). Furthermore, with the application of SDR, (16b) can be described as

$$
\theta \operatorname{Tr}\left(\boldsymbol{H}_{2} \boldsymbol{W}_{2}\right) \leq \operatorname{Tr}\left(\boldsymbol{H}_{2} \boldsymbol{W}_{1}\right)-\theta,
$$

where $\boldsymbol{H}_{i} \triangleq \boldsymbol{h}_{i} \boldsymbol{h}_{i}^{H}, i=1,2$.

Further, by introducing several auxiliary variables, i.e, $Q_{i}$, $r_{i}$ and $s_{i}, i=1,2$, the following correspondence to (12b) and (16a) can be shown

$$
\begin{gathered}
\boldsymbol{Q}_{1}=\boldsymbol{C}_{\mathbf{1}}^{\frac{1}{2}}\left(\boldsymbol{W}_{2}-\gamma \boldsymbol{W}_{1}\right) \boldsymbol{C}_{\mathbf{1}}^{\frac{1}{2}}, \\
\boldsymbol{r}_{1}=\boldsymbol{C}_{\mathbf{1}}^{\frac{1}{2}}\left(\boldsymbol{W}_{2}-\gamma \boldsymbol{W}_{1}\right) \tilde{\boldsymbol{h}}_{1}, \\
s_{1}=\tilde{\boldsymbol{h}}_{1}^{H}\left(\boldsymbol{W}_{2}-\gamma \boldsymbol{W}_{1}\right) \tilde{\boldsymbol{h}}_{1}-\frac{\gamma}{1-\beta}, \\
\boldsymbol{Q}_{2}=\boldsymbol{C}_{\mathbf{1}}^{\frac{1}{2}}\left(\boldsymbol{W}_{1}+\boldsymbol{W}_{2}\right) \boldsymbol{C}_{\mathbf{1}}^{\frac{1}{2}}, \\
\boldsymbol{r}_{2}=\boldsymbol{C}_{\mathbf{1}}^{\frac{1}{2}}\left(\boldsymbol{W}_{1}+\boldsymbol{W}_{2}\right) \tilde{\boldsymbol{h}}_{1}, \\
s_{2}=\tilde{\boldsymbol{h}}_{1}^{H}\left(\boldsymbol{W}_{1}+\boldsymbol{W}_{2}\right) \tilde{\boldsymbol{h}}_{1}-\frac{\gamma-\theta}{\beta|g|^{2}} .
\end{gathered}
$$

Finally, the original probabilistic outage constraint (12b) and the reformulated (16a) can be written as the following structure

$$
\operatorname{Pr}\left\{\boldsymbol{e}^{H} \boldsymbol{Q}_{i} \boldsymbol{e}+2 \operatorname{Re}\left\{\boldsymbol{e}^{H} \boldsymbol{r}_{i}\right\}+s_{i} \geq 0\right\} \geq 1-\rho_{i}, i=1,2 .
$$

\section{A. Bernstein-type inequality method}

To deal with a probabilistic constraint that has a form as (19), in this section we adopt the Bernstein-type inequality to make a convex approximation. Firstly, the following lemma is introduced which serves as a basis [14]:

Lemma 1: Let $\boldsymbol{e} \in \mathcal{C N}\left(0, \boldsymbol{I}_{\mathrm{n}}\right), \boldsymbol{Q} \in \mathbb{H}^{n}$ and $\boldsymbol{r} \in \mathbb{C}^{n}$. Then, for any $\varepsilon>0$, we have that

$$
\operatorname{Pr}\left\{\boldsymbol{e}^{\mathrm{H}} \boldsymbol{Q}_{\mathrm{i}} \boldsymbol{e}+2 \operatorname{Re}\left\{\boldsymbol{e}^{\mathrm{H}} \boldsymbol{r}_{\mathrm{i}}\right\} \geq \mathrm{T}(\varepsilon)\right\} \geq 1-\mathrm{e}^{-\varepsilon},
$$

where function $\mathrm{T}$ is defined as:

$$
\mathrm{T}(\varepsilon)=\operatorname{Tr}\left(\boldsymbol{Q}_{\mathrm{i}}\right)-\sqrt{2 \varepsilon} \sqrt{\left\|\boldsymbol{Q}_{\mathrm{i}}\right\|_{\mathrm{F}}^{2}+2\left\|\boldsymbol{r}_{\mathrm{i}}\right\|^{2}}-\varepsilon \lambda^{+}\left(\boldsymbol{Q}_{\mathrm{i}}\right),
$$

with $\lambda^{+}\left(\boldsymbol{Q}_{i}\right)=\max \left\{\lambda_{\max }\left(-\boldsymbol{Q}_{\mathrm{i}}\right), 0\right\}$ and $\lambda_{\max }\left(-\boldsymbol{Q}_{i}\right)$ be the maximum eigenvalue of $-\boldsymbol{Q}_{i}$.

The above inequality is the well-known Bernstein-type inequality, which can also be expressed by the inverse mapping $\mathrm{T}^{-1}$ as follows due to the monotonically decreasing characteristic of $\mathrm{T}(\varepsilon)$ :

$$
\operatorname{Pr}\left\{\boldsymbol{e}^{H} \boldsymbol{Q}_{i} \boldsymbol{e}+2 \operatorname{Re}\left\{\boldsymbol{e}^{H} \boldsymbol{r}_{i}\right\}+s_{i} \geq 0\right\} \geq 1-e^{-\mathrm{T}^{-1}(-\mathrm{s})} .
$$

It is easy to find that when $e^{-T^{-1}\left(-s_{i}\right)} \leq \rho_{i}$ holds, the inequality (22) is still satisfied if we replace $e^{-T^{-1}\left(-s_{i}\right)}$ with $\rho_{i}$. By adopting the Bernstain-type inequality and using the monotonically decreasing property of $\mathrm{T}$, we can obtain that

$$
\operatorname{Tr}\left(\boldsymbol{Q}_{\mathrm{i}}\right)-\sqrt{-2 \ln \left(\rho_{\mathrm{i}}\right)} \boldsymbol{o}+\ln \left(\rho_{\mathrm{i}}\right) \lambda^{+}\left(\boldsymbol{Q}_{\mathrm{i}}\right)+\mathrm{s}_{\mathrm{i}} \geq 0 .
$$

where $o=\sqrt{\left\|\boldsymbol{Q}_{i}\right\|_{F}^{2}+2\left\|\boldsymbol{r}_{i}\right\|^{2}}$. Furthermore, (23) can be reformulated as the following convex conic inequalities:

$$
\begin{gathered}
\operatorname{Tr}\left(\boldsymbol{Q}_{\mathrm{i}}\right)-\sqrt{-2 \ln \left(\rho_{\mathrm{i}}\right)} \mathrm{t}_{1}+\ln \left(\rho_{\mathrm{i}}\right) \mathrm{t}_{2}+\mathrm{s}_{\mathrm{i}} \geq 0, \\
\sqrt{\|\boldsymbol{Q}\|_{F}^{2}+2\left\|\boldsymbol{r}_{i}\right\|^{2}} \leq t_{1}, \\
t_{2} \boldsymbol{I}_{n}+\boldsymbol{Q}_{i} \succeq \mathbf{0}, \\
t_{2} \geq 0 .
\end{gathered}
$$

where $t 1, t 2 \in \mathbb{R}$ are two introduced auxiliary variables.

Hence, one can note that the probabilistic inequality (19) is transformed into efficiently computable convex restrictions as (24a)-(24d). Finally, by applying the SDR approach and Bernstein-type method, problem P1 is reformulated as

$$
\begin{aligned}
\mathrm{P} 2: \max _{\beta, \boldsymbol{W}_{1}, \boldsymbol{W}_{2}} & (1-\beta) \operatorname{Tr}\left(\boldsymbol{H}_{1} \boldsymbol{W}_{1}\right) \\
\text { s.t. } \quad & \operatorname{Tr}\left(\boldsymbol{Q}_{1}\right)-\sqrt{-2 \ln \left(\rho_{1}\right)} t_{1}+\ln \left(\rho_{1}\right) t_{2}+s_{1} \geq 0, \\
& \sqrt{\left\|\boldsymbol{Q}_{1}\right\|_{F}^{2}+2\left\|\boldsymbol{r}_{1}\right\|^{2}} \leq t_{1}, \\
& t_{2} \boldsymbol{I}_{n}+\boldsymbol{Q}_{1} \succeq \mathbf{0}, \\
\operatorname{Tr}\left(\boldsymbol{Q}_{2}\right)- & \sqrt{-2 \ln \left(\rho_{2}\right)} \mathrm{t}_{3}+\ln \left(\rho_{2}\right) \mathrm{t}_{4}+\mathrm{s}_{2} \geq 0, \\
& \sqrt{\left\|\boldsymbol{Q}_{2}\right\|_{F}^{2}+2\left\|\boldsymbol{r}_{2}\right\|^{2}} \leq t_{3}, \\
& t_{4} \boldsymbol{I}_{n}+\boldsymbol{Q}_{2} \succeq \mathbf{0} \\
& t_{2} \geq 0, t_{4} \geq 0 \\
& \theta \operatorname{Tr}\left(\boldsymbol{H}_{2} \boldsymbol{W}_{2}\right) \leq \operatorname{Tr}\left(\boldsymbol{H}_{2} \boldsymbol{W}_{1}\right)-\theta, \\
& \operatorname{Tr}\left(\boldsymbol{H}_{1} \boldsymbol{W}_{2}\right) \geq \operatorname{Tr}\left(\boldsymbol{H}_{1} \boldsymbol{W}_{1}\right), \\
& \operatorname{Tr}\left(\boldsymbol{H}_{2} \boldsymbol{W}_{2}\right) \geq \operatorname{Tr}\left(\boldsymbol{H}_{2} \boldsymbol{W}_{1}\right), \\
& \operatorname{Tr}\left(\boldsymbol{W}_{1}\right)+\operatorname{Tr}\left(\boldsymbol{W}_{2}\right) \leq P_{\max }, \\
& 0 \leq \beta \leq 1,
\end{aligned}
$$

where $Q_{i}, r_{i}$ and $s_{i}, i=1,2$, are defined as (18a)-(18f).

Remark 1: The optimal solution to problem $\mathrm{P} 2$ can be obtained through two-dimensional exhaustive search of variables $\beta$ and $\theta$.

However, the complexity is too high for two-dimensional exhaustive search, which motivates us to find a low-complexity suboptimal solution with the help of SCA and arithmetic geometric mean (AGM) in the next subsection. 


\section{B. SCA-based transformation}

By applying epigraph reformulation and introducing two auxiliary variables $\mu$ an $\nu$, the objective function (25a) can be recast as:

$$
\begin{aligned}
& \max \quad \mathrm{u} \\
& \text { s.t. } \nu^{2} \geq \mu, \\
& {\left[\begin{array}{cc}
1-\beta & \nu \\
\nu & \operatorname{Tr}\left(\boldsymbol{H}_{1} \boldsymbol{W}_{1}\right)
\end{array}\right] \succeq 0 .}
\end{aligned}
$$

Hence, (25a) is converted into a linear objective function of (26a), a convex linear matrix inequality (LMI) of (26c) and a nonconvex quadratic inequality (26b).

To approximate (26b), we first introduce a convex lower bound for $\nu^{2}$ by applying the first-order Taylor approximation, i.e.,

$$
\nu^{2} \geq 2 \nu^{(n)} \nu-\left(\nu^{(n)}\right)^{2},
$$

where $\nu^{(n)}$ denotes the value of $\nu$ at iteration $n$. By replacing $\nu^{2}$ with the inequality (27), (26b) can then be approximated by a convex constraint, which is given as

$$
2 \nu^{(n)} \nu-\left(\nu^{(n)}\right)^{2} \geq \mu \text {. }
$$

In addition, by applying the AGM method, we can approximate the constraint $(25 \mathrm{i})$ using the following convex function:

$$
\left(a_{1}^{(n)} \theta\right)^{2}+\left(\operatorname{Tr}\left(\boldsymbol{H}_{2} \boldsymbol{W}_{1}\right) / a_{1}^{(n)}\right)^{2} \leq 2 \operatorname{Tr}\left(\boldsymbol{H}_{2} \boldsymbol{W}_{2}\right)-2 \theta
$$

where the setting of $a_{1}^{(n)}$ can be given by

$$
a_{1}^{(n)}=\sqrt{\left(\operatorname{Tr}\left(\boldsymbol{H}_{2}^{H} \boldsymbol{W}_{1}\right)^{(n-1)} / \theta^{(n-1)}\right.} .
$$

Now the remaining problem lies in $(25 \mathrm{e})$, as the formation of $s_{2}$ in $(25 \mathrm{e})$ is nonconvex. By introducing a slack variable $\xi$, we can reformulate $s_{2}$ as

$$
\begin{gathered}
s_{2}=\tilde{\boldsymbol{h}}_{1}^{H}\left(\boldsymbol{W}_{1}+\boldsymbol{W}_{2}\right) \tilde{\boldsymbol{h}}_{1}-\frac{\gamma}{\beta|g|^{2}}+\xi, \\
\left(a_{2}^{(n)} \beta\right)^{2}+\left(\xi / a_{2}^{(n)}\right)^{2} \leq \frac{2 \theta}{|g|^{2}},
\end{gathered}
$$

where $a_{2}^{(n)}=\sqrt{\xi_{2}^{(n-1)} / \beta^{(n-1)}}$. Here, (31b) is obtained with the AGM-inequality method and the transformation process is omitted.

With the above proposed approximation methods, the original problem can be transformed to a convex program. During iteration $n$, we need to solve the following convex optimization problem:

$$
\begin{gathered}
\text { P3 : } \max _{\mu, \nu, \beta, \boldsymbol{W}_{1}, \boldsymbol{W}_{2}} \mu \\
\text { s.t. } 2 \nu^{(n)} \nu-\left(\nu^{(n)}\right)^{2} \geq \mu \\
{\left[\begin{array}{cc}
1-\beta & \nu \\
\nu & \operatorname{Tr}\left(\boldsymbol{H}_{1} \boldsymbol{W}_{1}\right)
\end{array}\right] \succeq 0} \\
(25 \mathrm{~b}),(25 \mathrm{c}),(25 \mathrm{~d}),(25 \mathrm{e}),(25 \mathrm{f}),(25 \mathrm{~g}), \\
(25 \mathrm{~h}),(25 \mathrm{j}),(25 \mathrm{k}),(25 \mathrm{l}),(29),(31 \mathrm{a}),(31 \mathrm{~b}) .
\end{gathered}
$$

Finally, to solve the problem P3, we provide the SCA-based iterative method, outlined as Algorithm 1.

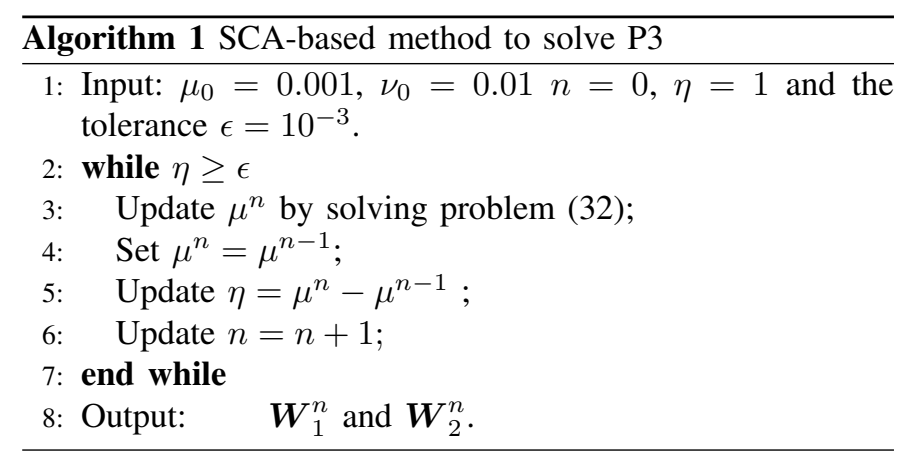

\section{NumericAl RESUlTs}

In this section, we provide Monte Carlo simulation results to evaluate the performance of the proposed robust algorithm for a SWIPT-enabled cooperative NOMA system. We iteratively solve the robust optimization problem for 2,000 times. It is assumed that the BS has two antennas, i.e. $N_{t}=2$, while user 1 and user 2 each has one. The estimated channel coefficient can be modeled as $\tilde{\boldsymbol{h}}_{k}=\tilde{\boldsymbol{g}}_{k} d_{k}^{-\frac{\alpha}{2}}, k=\{1,2\}$, where $d_{k}$ is the distance from the BS to the $k$-th user, $\alpha$ is the path loss exponent. We set $\alpha=2.5$ and $\tilde{\boldsymbol{g}}_{k}$ follows Rayleigh fading distribution. Without loss of generality, the bandwidth is set to be $1 \mathrm{MHz}$. All the background noise power is assumed to be 1 Watt, and the transmit power is shown in $\mathrm{dB}$. Furthermore, in order to provide comprehensive studies, the noncooperative NOMA and TDMA schemes, with robust and non-robust beamforming design, are all introduced to compare with the proposed robust cooperative NOMA scheme.

We firstly provide insight on the convergence property of the proposed algorithm. As can be observed from Fig. 2, it indicates that Algorithm 1 can converge to the maximum value within 3 iterations, which proves the effectiveness of the proposed algorithm.

In Fig. 3, the impact of the error variance is shown. Specifically, we set error covariances $C_{1}$ and $C_{2}$ be the same value as $\epsilon_{h}^{2}$, the desired data rate of user 2 as $1 \mathrm{Mbps}$, the available maximum power at the $B S$ be $20 \mathrm{~dB}$, and the outage is set to be 0.1 which means that the system has a chance of $90 \%$ or higher probability to satisfy the SINR requirements. The figure illustrates that the proposed SCAbased Algorithm 1 obtains similar performance as exhaustive search method, but has significantly reduced computational complexity. Furthermore, we can observe that although the user 1's maximum achievable rate decreases for all of the schemes when the error variance becomes larger, the benefit of using the proposed SWIPT-enabled robust cooperative NOMA scheme becomes more significant since the difference gap between the proposed model and the other two schemes becomes bigger. Moreover, both of the NOMA schemes illustrated in this figure yield better performance than TDMA which shows the advantage of applying NOMA in the outage-based robust transmit beamforming design.

To thoroughly investigate the performance of the proposed system model, Fig. 4 illustrates the maximum achievable rate of user 1 versus the available transmission power at the BS 


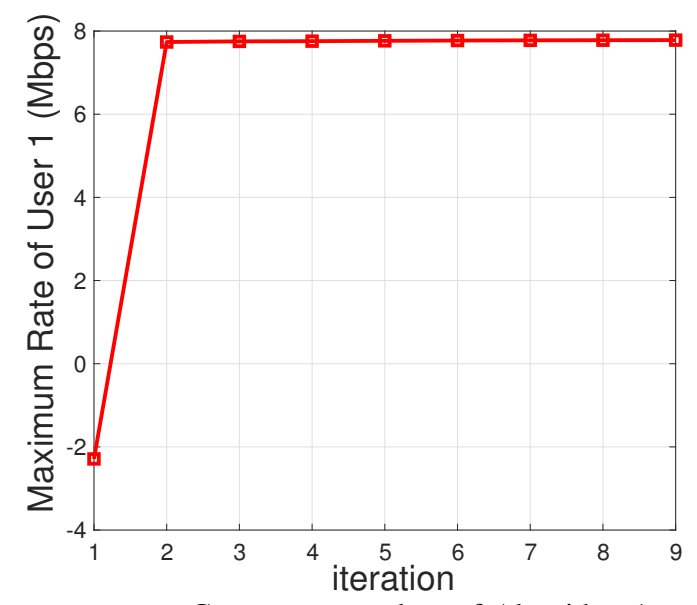

Fig. 2. Converge procedure of Algorithm 1.

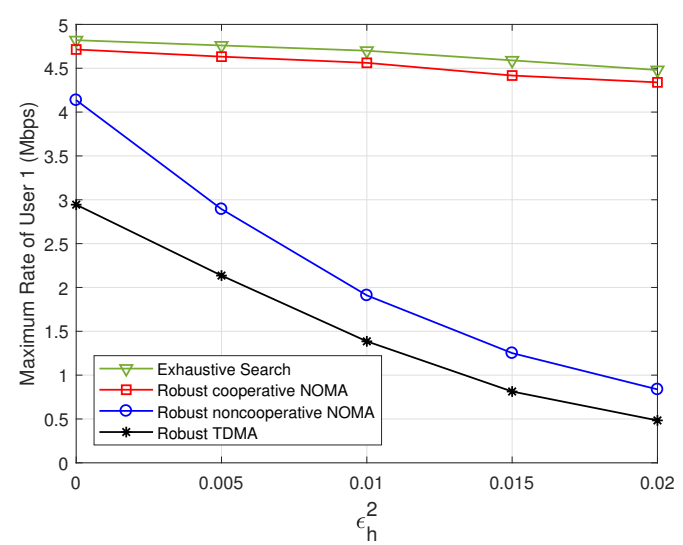

Fig. 3. Achievable rate of user 1 vs. error variance with $\gamma=1$.

for the following schemes: the proposed robust cooperative NOMA, cooperative NOMA with perfect CSI, robust noncooperative NOMA, noncooperative NOMA with perfect CSI, classical robust TDMA and TDMA with perfect CSI. First, it demonstrates that when perfect CSI is available at the BS, cooperative NOMA outperforms noncooperative NOMA when the transmit power is small and achieves the same data rate when transmit power excesses $20 \mathrm{~dB}$. Moreover, Fig. 4 indicates that the proposed robust cooperative NOMA system always achieves better performance than the robust noncooperative NOMA and TDMA, which means that it is beneficial to adopt the cooperative transmission design for the scenarios with only imperfect CSI available.

\section{CONClusion}

In this paper, we investigated the robust transmit beamforming and PS design to maximize the strong user's achievable data rate for a SWIPT-enabled cooperative NOMA system with channel uncertainties considered. The formulated problem was first transformed into a more tractable form with the SDR technique. Specifically, the Bernstein-type inequality was applied to transform the probabilistic constraints into manageable and computable approximations that can be globally solved by two-dimensional exhaustive search. Finally, an iterative low-

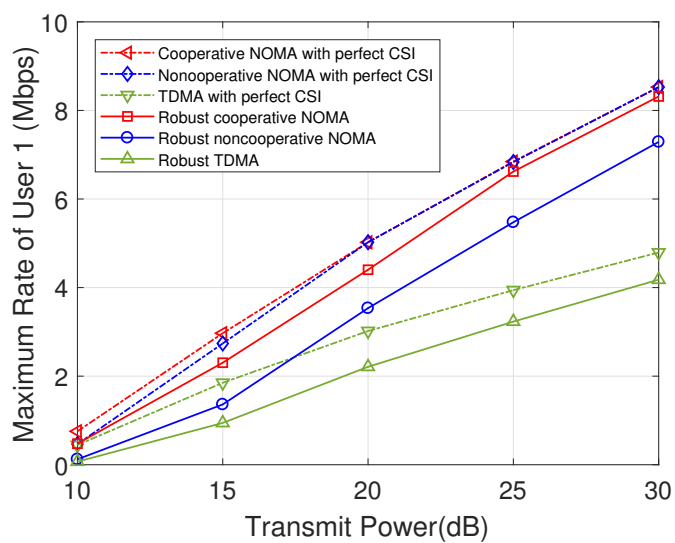

Fig. 4. Achievable rate of user 1 vs. transmit power with $\gamma=1$.

complexity algorithm was developed to reduce the high complexity, which provided near-optimal performance. Numerical results proved the superiority of the proposed SWIPT-enabled cooperative NOMA design over other schemes.

\section{REFERENCES}

[1] W. Yu, L. Musavian, and Q. Ni, "Link-layer capacity of NOMA under statistical delay QoS guarantees," IEEE Trans. Commun., vol. 66, no. 10, pp. 4907-4922, Oct. 2018.

[2] Z. Qin, X. Yue, Y. Liu, Z. Ding, and A. Nallanathan, "User association and resource allocation in unified NOMA enabled heterogeneous ultra dense networks," IEEE Commun. Mag., vol. 56, no. 6, pp. 86-92, Jun. 2018.

[3] L. Lv, J. Chen, et al., "Cooperative non-orthogonal multiple access in cognitive radio," IEEE Commun. Lett., vol. 20, no. 10, pp. 2059-2062, Oct. 2016.

[4] J. Ye, H. Lei, Y. Liu, G. Pan, et al., "Cooperative communications with wireless energy harvesting over Nakagami- $m$ fading channels," IEEE Trans. Commun., vol. 65, no. 12, pp. 5149-5164, Dec. 2017.

[5] Y. Liu, Z. Ding, M. Elkashlan, and H. V. Poor, "Cooperative nonorthogonal multiple access with simultaneous wireless information and power transfer,' IEEE J. Sel. Areas Commun., vol. 34, no. 4, pp. 938953, Apr. 2016.

[6] Y. Xu, C. Shen, Z. Ding, X. Sun, S. Yan, G. Zhu, and Z. Zhong, "Joint beamforming and power-splitting control in downlink cooperative SWIPT NOMA systems," IEEE Trans. Signal Process., vol. 65, no. 18, pp. 4874-4886, Sept. 2017.

[7] Y. Hao, Q. Ni, H. Li, and S. Hou, "Robust multi-objective optimization for EE-SE tradeoff in D2D communications underlaying heterogeneous networks," IEEE Trans. Commun., vol. 66, no. 10, pp. 4936-4949, Oct. 2018.

[8] Y. Sun, D. W. K. Ng, J. Zhu, and R. Schober, "Robust and secure resource allocation for full-duplex MISO multicarrier NOMA systems," arXiv preprint arXiv:1710.01391, 2017.

[9] C. C. Zarakovitis, Q. Ni, D. Skordoulis, and M. G. Hadjinicolaou, "Power-efficient cross-layer design for OFDMA systems with heterogeneous QoS, imperfect CSI, and outage considerations," IEEE Trans. Veh. Technol., vol. 61, no. 2, pp. 781-798, Feb. 2012.

[10] L. Liu, R. Zhang, and K.-C. Chua, "Multi-antenna wireless powered communication with energy beamforming," IEEE Trans. Commun., vol. 62, no. 12 , pp. 4349-4361, Dec. 2014.

[11] M. F. Hanif, Z. Ding, T. Ratnarajah, and G. K. Karagiannidis, "A minorization-maximization method for optimizing sum rate in the downlink of non-orthogonal multiple access systems," IEEE Trans. Signal Process., vol. 64, no. 1, pp. 76-88, Sept. 2016.

[12] K.-Y. Wang, A. M.-C. So, T.-H. Chang, W.-K. Ma, and C.-Y. Chi, "Outage constrained robust transmit optimization for multiuser MISO downlinks: Tractable approximations by conic optimization," IEEE Trans. Signal Process., vol. 62, no. 21, pp. 5690-5705, Nov. 2014.

[13] I. Bechar, "A bernstein-type inequality for stochastic processes of quadratic forms of Gaussian variables," arXiv preprint arXiv:0909.3595, 2009. 